\title{
MicroRNA profiles in five pairs of early gastric cancer tissues and adjacent non-cancerous tissues
}

\author{
YUAN LIANG ${ }^{1-3^{*}}$, YONGXUN ZHAO $^{4^{*}}$, LONGQUAN LI $^{1-3^{*}}$, HUI WEI $^{1-3}$, TAOBI HUANG $^{1-3}$, \\ HUIYUN ZHANG ${ }^{1-3}$, XIA CHEN ${ }^{1-3}$, HENG YUN ${ }^{5}$, WEIMING SUN ${ }^{6}$ and YUPING WANG ${ }^{2,3}$ \\ ${ }^{1}$ The First School of Clinical Medicine, Lanzhou University; ${ }^{2}$ Department of Gastroenterology, \\ The First Hospital of Lanzhou University; ${ }^{3}$ Key Laboratory for Gastrointestinal Diseases of Gansu Province, \\ The First Hospital of Lanzhou University; ${ }^{4}$ Department of Oncology Surgery, \\ The First Hospital of Lanzhou University, Lanzhou, Gansu 730000; ${ }^{5}$ Department of General Surgery, \\ Baiyin First People's Hospital, Baiyin, Gansu 730900; ${ }^{6}$ Department of Endocrinology, \\ The First Hospital of Lanzhou University, Lanzhou, Gansu 730000, P.R. China
}

Received November 10, 2020; Accepted April 13, 2021

DOI: 10.3892/ol.2021.12856

\begin{abstract}
Approximately half of the world's gastric cancer cases and deaths occur in China. In addition, the incidence and mortality rates of gastric cancer in Gansu province in China are much higher than the average nationwide levels. The present study investigated microRNA (miRNA/miR) profiles in early gastric cancer (EGC) without specific symptoms. miRNA expression levels in five pairs of EGC tissues and adjacent non-cancerous mucosa tissues of patients from Gansu province in China were analyzed using a miRNA microarray. A total of 47 differentially expressed miRNAs (DEMs) were identified. Subsequently, mRNA expression profiles of three pairs of cancer tissues and adjacent non-cancerous tissues from 3 Asian patients with stage I or stage II gastric cancer (stage I/II; American Joint Committee on Cancer classification, Eighth Edition) were obtained from The Cancer Genome Atlas database, and differentially expressed genes (DEGs) were identified. The target genes of DEMs were filtered from the DEGs using the miRDB database and a miRNA-gene network was constructed. The functions of DEMs were evaluated using the tool for annotations of human miRNAs database,
\end{abstract}

Correspondence to: Dr Yuping Wang, Department of Gastroenterology, The First Hospital of Lanzhou University, 1 West Donggang Road, Lanzhou, Gansu 730000, P.R. China

E-mail: wangyuping@lzu.edu.cn

Dr Weiming Sun, Department of Endocrinology, The First Hospital of Lanzhou University, 1 West Donggang Road, Lanzhou, Gansu 730000, P.R. China

E-mail: swm77@163.com

*Contributed equally

Key words: early gastric cancer, microRNA, microarray, The Cancer Genome Atlas, Gansu province, China and via Gene Ontology analysis, Kyoto Encyclopedia of Genes and Genomes analysis and Gene Set Enrichment Analysis of the target genes. Finally, survival analyses of DEMs, which were in the miRNA-gene network, was performed. The results suggested that a number of miRNAs, including hsa-let-7a-5p, hsa-miR-27a-3p, hsa-miR-126-5p and hsa-miR-424-5p, may serve critical roles in EGC. The present study could provide a basis for the identification of EGC screening biomarkers. Furthermore, the present study may provide a basis for the exploration of the cause of the high incidence of gastric cancer in Gansu province from the perspective of miRNAs.

\section{Introduction}

Gastric cancer was the third most common cause of cancer-associated mortality in Asia in 2018 (1). Asia has a high incidence of gastric cancer globally, and approximately half of the world's gastric cancer cases and deaths occur in China (1). In China, gastric cancer is the second most common malignancy and the third leading cause of death from malignant neoplasms (1). In 2012, Gansu province in China had a gastric cancer age-standardized incidence rate by Chinese standard population and a mortality rate of 62.34/100,000 and $36.94 / 100,000$ (2), respectively, which is much higher than the average levels in China of 22.06/100,000 and 15.16/100,000 (3). The prevalence of gastric cancer in Wuwei, Gansu, China is almost five times higher than that nationwide (4). Our previous study established a large-scale natural population cohort involving 24,115 individuals from Wuwei, Gansu, China, and has conducted research regarding various aspects to explore the causes of the high incidence of gastric cancer in this region and to provide a theoretical basis for the formulation of control policies (5).

Great efforts, such as the development of more effective biomarkers for diagnosis, prognosis, monitoring and prediction, have been made regarding the clinical management of patients with gastric cancer (6). Gastric cancer has atypical symptoms in the early stage and lacks effective early screening 
markers; therefore, most patients have entered the advanced stage when they are detected (7). A retrospective study revealed that the 5-year rate of relative survival of patients with early gastric cancer (EGC) with treatment is $105.0 \%$ compared with the expected survival of individuals from the general population matched for age and sex (8). By contrast, few EGCs are discovered in China and the West, leading to 5-year relative survival rates of $10-40 \%$ (9-11). In Japan, the male mortality rate of gastric cancer has fallen by more than half since a mass screening program was introduced in the early 1970s (9). Therefore, early diagnosis and treatment of gastric cancer, and screening are important. Gastroscopy is a valuable tool for reducing the mortality associated with gastric cancer (12). However, due to its acceptability and cost, large-scale screening and early detection with gastroscopy might not be easy. At present, available tumor markers for gastric cancer, including carcinoembryonic antigen and cancer antigen 19-9, are useful for detecting recurrence and distant metastasis or predicting patient survival; however, these are inadequate for gastric cancer screening due to their low sensitivity, particularly for EGC (13). Therefore, there is an urgent requirement for novel non-invasive methods for the screening of patients with gastric cancer, and microRNAs (miRNAs/miRs) have been increasingly studied for this.

miRNAs are endogenous 18-24 nucleotide RNAs, which can serve critical regulatory roles in animals and plants (14). miRNAs can combine with other associated proteins to form an active RNA-induced silencing complex (RISC), and RISC combines with the 5' untranslated region, open reading frames or $3^{\prime}$ untranslated region of a target gene mRNA to suppress its translation or to induce its degradation $(15,16)$. An increasing number of studies have reported that miRNAs can be used as biomarkers for gastric cancer diagnosis, as well as targets for disease treatment (17-19). For example, a study of 682 participants examined the expression levels of 578 miRNAs in serum and demonstrated that the combination of 12 miRNAs in serum has excellent diagnostic value for gastric cancer (13). Microarray technology has been widely used to investigate miRNA expression in multiple tumor types, such as gastric cancer and lung cancer $(20,21)$.

The present study, as a part of the aforementioned Wuwei cohort research project, utilized samples collected from patients diagnosed with EGC during the screening of this disease in Wuwei. miRNA profiles in five pairs of EGC tissues and adjacent non-cancerous tissues were explored using a miRNA microarray. Bioinformatics methods were used to analyze the functions and mechanisms of the dysregulated miRNAs, as well as their potential as prognostic factors. The present study aimed to provide a basis for the identification of EGC screening biomarkers and to discuss the particularity of gastric cancer in Gansu province from the perspective of miRNAs.

\section{Materials and methods}

Patients and samples. EGC tissues and adjacent non-cancerous mucosa tissues (5 $\mathrm{cm}$ from the cancer tissues) were obtained from 5 patients with EGC who were treated with endoscopic submucosal dissection at the Department of Gastroenterology, The Gansu Wuwei Tumor Hospital (Wuwei, China) and
Department of Gastroenterology, The First Hospital of Lanzhou University (Lanzhou, China) between November 2014 and April 2015. The inclusion criteria were: i) Patients who agreed to participate in the study and signed the informed consent were included; ii) all patients were pathologically diagnosed as EGC. The tumor stage was confirmed according to the Eighth Edition of the The American Joint Committee on Cancer (AJCC) TNM staging system guidelines (22); and iii) the patients did not receive radiotherapy or chemotherapy before surgery. The exclusion criteria were: i) Patients who did not agree to participate in the study; ii) patients who had other malignancies; and iii) patients who had other diseases, such as diabetes mellitus and hypertension. The patients included 3 men and 2 women. Clinicopathological features of the patients are listed in Table I. The present study was approved by the Ethics Committee of The First Hospital of Lanzhou University (approval no. LDYYLL2012001; Lanzhou, China). All patients and their family members signed an informed consent form. The tissue samples were stored and transported at $-80^{\circ} \mathrm{C}$.

RNA extraction. Total RNA from 10 tissue samples was isolated using TRIzol ${ }^{\circledR}$ (Invitrogen; Thermo Fisher Scientific, Inc.) and purified using a RNeasy mini kit (Qiagen, Inc.) according to the manufacturer's protocols. RNA quality and quantity were measured using a NanoDrop spectrophotometer (ND-1000; NanoDrop Technologies; Thermo Fisher Scientific, Inc.). RNA integrity was determined by formaldehyde agarose gel electrophoresis in 3-(N-morpholino)propanesulfonic acid buffer with a $1.2 \%$ gel. Ethidium bromide was used as a fluorescent dye, and the GelDoc Go Gel Imaging System (Bio-Rad Laboratories, Inc.) was used for imaging.

miRNA labeling and array hybridization. miRNA microarray assays were performed by Aksomics, Inc. The miRCURY TM Hy $3^{\mathrm{TM}} / \mathrm{Hy}^{\mathrm{TM}}$ Power labeling kit (Exiqon; Qiagen, Inc.) was used with total RNA samples according to the manufacturer's guidelines for miRNA labeling. After termination of the labeling procedure, the $\mathrm{Hy} 3^{\mathrm{TM}}$-labeled samples were hybridized on the array in miRCURY LNA ${ }^{\mathrm{TM}}$ microRNA Array Kit, 7th generation-hsa, mmu and rno (Exiqon; Qiagen, Inc.). Following hybridization, the slides were washed several times using Wash buffer kit (Exiqon; Qiagen, Inc.). The slides were scanned using an Axon GenePix 4000 B microarray scanner (Molecular Devices, LLC).

Microarray data processing. Scanned images were subsequently imported into GenePix Pro 6.0 (Molecular Devices, LLC) for grid alignment and data extraction. Data analyses were performed using R software (v3.6.3; https://cran.r-project. org/src/base/R-3/). The 'limma' package (v3.42.2) (23) in $\mathrm{R}$ was used for background correction and normalization between arrays. The robust multi-array average algorithm was selected when performing background correction, and the 'offset' parameter was set to 50. Expression data were normalized using median normalization. The landing lights (probe ID 13138; annotated as $\mathrm{Hy} 3^{\mathrm{TM}}$ ) were only included for gal-file orientation, and their corresponding data points were removed prior to the normalization of the dataset, according to the manual of the miRCURY LNA ${ }^{\mathrm{TM}}$ microRNA Array Kit, 7th 
Table I. Clinicopathological features of patients with early gastric cancer whose tissues underwent microRNA microarray testing.

\begin{tabular}{|c|c|c|c|c|c|c|c|c|c|}
\hline Patient & Sex & $\begin{array}{l}\text { Age, } \\
\text { years }\end{array}$ & Location & Lesion location & Histology & T stage & $\begin{array}{c}\mathrm{N} \\
\text { stage }\end{array}$ & $\begin{array}{c}\text { M } \\
\text { stage }\end{array}$ & $\begin{array}{c}\text { AJCC } \\
\text { pathologic stage } \\
\text { (8th edition) }\end{array}$ \\
\hline Patient 1 & Female & 65 & Wuwei, Gansu, China & Cardia & Adenocarcinoma & T1a & 0 & 0 & IA \\
\hline Patient 2 & Male & 72 & Wuwei, Gansu, China & Gastric antrum & Adenocarcinoma & Tis & 0 & 0 & 0 \\
\hline Patient 3 & Male & 46 & Wuwei, Gansu, China & Gastric body & Adenocarcinoma & $\mathrm{T} 1 \mathrm{~b}$ & 0 & 0 & IA \\
\hline Patient 4 & Male & 45 & Wuwei, Gansu, China & Cardia & Adenocarcinoma & T1a & 0 & 0 & IA \\
\hline Patient 5 & Female & 67 & Lanzhou, Gansu, China & Gastric antrum & Adenocarcinoma & Tis & 0 & 0 & 0 \\
\hline
\end{tabular}

AJCC, American Joint Committee on Cancer.

generation-hsa, mmu and rno (Exiqon; Qiagen, Inc.). For the same probes, the expression data of all samples corresponding to each probe were averaged. The probe with the maximum average value was retained among the same probes. The 'limma' package was used to give a microarray linear model fit, compute moderated t-statistics, moderated F-statistic, and log-odds of differential expression by empirical Bayes moderation of the standard errors towards a common value. The cutoff criteria for differentially expressed miRNAs (DEMs) were a $\mid \log _{2}$ fold change $\left(\log _{2} \mathrm{FC}\right) \mid \geq 1$ and an adjusted P-value (adj. P. val) $<0.05$. Data were submitted to the Gene Expression Omnibus database (24) (dataset accession no. GSE158315).

Functional annotation of DEMs. The functions of DEMs were annotated using TAM (v2.0), a web-based program for annotations of human miRNAs (25).

mRNA expression profiles of gastric cancer at stage I/II based on data from The Cancer Genome Atlas (TCGA). The mRNA expression profiles of three pairs of gastric cancer tissues and their matched adjacent non-cancerous mucosa tissues were obtained from TCGA [project ID, TCGA-stomach adenocarcinoma (STAD)] using the Genomic Data Commons Data Transfer Tool (v1.5.0; https://gdc. cancer.gov/access-data/gdc-data-transfer-tool). The samples were obtained from 3 Asian patients with stage I/II gastric cancer (AJCC, Eighth Edition; Table II). The 'DESeq2' package (v1.26.0; http://www.bioconductor.org/packages/release/bioc/html/DESeq2.html) (26) in R was used to perform the differential analysis. Differentially expressed genes (DEGs) with $\log _{2} \mathrm{FCl} \geq 2$ and adj. $\mathrm{P}$. val $<0.05$ in cancer tissues compared with non-cancerous tissues were filtered.

Functional enrichment and pathway analysis of DEGs. Gene Ontology (GO) analysis (27), Kyoto Encyclopedia of Genes and Genomes (KEGG) analysis (28) and Gene Set Enrichment Analysis (GSEA) (29) were performed on the DEGs in the cancer tissues and adjacent non-cancerous tissues of 3 Asian patients with stage I/II gastric cancer. First, the 'org.Hs.eg.db' package (v3.10.0; http://www. bioconductor.org/packages/release/data/annotation/html/org. Hs.eg.db.html) was used to convert the gene symbols into entrezIDs. Subsequently, 'clusterProfiler' (v3.14.3; http://www. bioconductor.org/packages/release/bioc/html/clusterProfiler. html) (30), 'ggplot2' (v3.3.2) (31) and 'enrichplot' (v1.6.1; http://bioconductor.org/packages/devel/bioc/html/enrichplot. html) packages were utilized to find the enriched GO terms and KEGG pathways, where a false discovery rate $<0.05$ was considered to be statistically significant. A 'gmt' format file called 'Hallmark gene sets, NCBI (Entrez) gene IDs', which includes the information of the gene sets and their associated pathways, was downloaded from the GSEA website (https://www.gsea-msigdb.org/gsea/index.jsp) and was used to perform GSEA in R software. GO and KEGG analyses of the predicted DEM target genes were performed in the same manner.

Prediction of DEM target genes. The miRDB database (http://mirdb.org/), an online database for miRNA target prediction and functional annotation, was used to predict the target relationships between DEMs and DEGs (32). DEMs and DEGs were controlled to have opposite trends in expression level variations between cancer tissues and non-cancerous tissues. In other words, for the upregulated DEMs, target genes were predicted among the downregulated DEGs and vice versa. Subsequently, a miRNA-gene regulatory network was constructed and visualized using Cytoscape software (v3.7.2) (33).

Construction of the target gene protein-protein interaction (PPI) network and hub genes association network. The target genes were input into the Search Tool for the Retrieval of Interacting Genes/Proteins database (v11.0; https://string-db. org) (34), an online protein interaction search tool for the retrieval of interacting genes/proteins, to construct a PPI network. 'Experiments', 'Databases', 'Co-experiment' and 'Co-occurrence' were set as the parameter of 'active interaction sources', and the interactions with a combined score $>0.7$ were selected for the PPI network. The PPI network was visualized using Cytoscape software, and the tight link hub mRNAs within the PPI network were calculated using Molecular Complex Detection in Cytoscape (v2.0.0; http://apps.cytoscape.org/apps/mcode) (35) with default parameters.

Survival analysis validation. To verify the results obtained, clinical data and miRNA expression data of 80 Asian patients with gastric cancer were downloaded from TCGA (project ID, TCGA-STAD). For each miRNA, which was in 
Table II. Clinicopathological features of patients with gastric cancer at stage I/II whose gene expression profiles were obtained from TCGA.

\begin{tabular}{|c|c|c|c|c|c|c|c|c|c|}
\hline Patient & Sex & $\begin{array}{l}\text { Age, } \\
\text { years }\end{array}$ & Ethnicity & Lesion location & Histology & $\begin{array}{c}\mathrm{T} \\
\text { stage }\end{array}$ & $\begin{array}{c}\mathrm{N} \\
\text { stage }\end{array}$ & $\begin{array}{c}\mathrm{M} \\
\text { stage }\end{array}$ & $\begin{array}{c}\text { AJCC } \\
\text { pathologic stage } \\
\text { (8th Edition) }\end{array}$ \\
\hline TCGA-HU-A4GH & Male & 75 & Asian & Gastric body & Adenocarcinoma & $\mathrm{T} 1 \mathrm{~b}$ & No & M0 & IA \\
\hline TCGA-HU-A4GP & Female & 62 & Asian & Gastric antrum & Adenocarcinoma & $\mathrm{T} 2$ & N1 & M0 & IIA \\
\hline TCGA-HU-A4HB & Male & 68 & Asian & Gastric antrum & Adenocarcinoma & $\mathrm{T} 2$ & $\mathrm{~N} 2$ & M0 & IIB \\
\hline
\end{tabular}

AJCC, American Joint Committee on Cancer; TCGA, The Cancer Genome Atlas.

the miRNA-gene network, the 'survminer' package (v0.4.8; https://cran.r-project.org/web/packages/survminer/index.html) was used to find a best separation cutoff value, and then patients were divided into two groups according to the cutoff value. The prognostic values of partial DEMs were identified by Kaplan-Meier analysis with the 'survival' package (v3.2.3) (36) in $\mathrm{R}$ software, and $\mathrm{P}<0.05$ (log-rank test) was considered to indicate a statistically significant difference.

Statistical analysis. The statistical analyses were performed using R software. A moderated t-test was performed to screen the DEMs with the 'limma' package, and a paired samples t-test was performed to screen the DEGs with the 'DESeq2' package. The expression levels of miRNAs and genes are presented as $\log _{2}$ FC. The log-rank test was performed in the survival analysis with the 'survival' package. $\mathrm{P}<0.05$ was considered to indicate a statistically significant difference.

\section{Results}

Microarray data processing and identification of DEMs. A flowchart of the research design is shown in Fig. 1. The box and density plots indicated the normalization results of miRNA microarray data for 10 tissue samples (Fig. 2A and B). A total of seven upregulated and 40 downregulated human miRNAs were identified using the criteria of $\log _{2} \mathrm{FC} \geq 1$ and adj. P. val $<0.05$ [seven downregulated miRNAs of human viruses and one downregulated small nuclear RNA (snRNA) were also detected]. The heatmap demonstrated that the samples could be separated into two groups based on the 55 dysregulated RNA molecules (Fig. 2C). The volcano plot revealed the expression distribution of miRNAs (Fig. 2D), and the principal component analysis plot is shown in Fig. $2 \mathrm{E}$.

Functional annotations of DEMs. The functions of the DEMs were annotated using TAM. 'Hormones regulation', 'Human embryonic stem cell (hESC) regulation', 'Immune response', 'Inflammation', 'Cell cycle related', 'Epithelial-mesenchymal transition', 'Cell death', 'Hematopoiesis' and 'MiRNA tumor suppressors' were the most enriched function terms (Table III).

Identification of DEGs using samples from TCGA. To further investigate the functions of DEMs via their target genes, the present study aimed to obtain DEGs between EGC and non-cancerous tissues. Since there was only 1 Asian patient

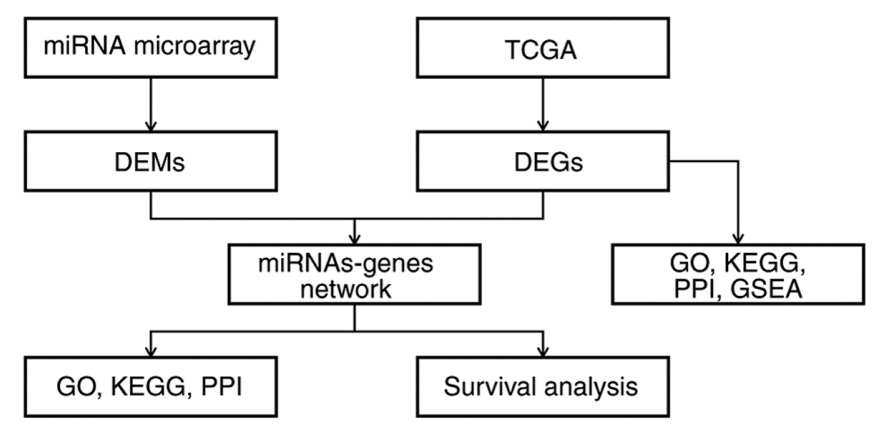

Figure 1. Flowchart of the study design. DEGs, differentially expressed genes; DEMs, differentially expressed miRNAs; GO, Gene Ontology; GSEA, Gene Set Enrichment Analysis; KEGG, Kyoto Encyclopedia of Genes and Genomes; miRNA, microRNA; PPI, protein-protein interaction; TCGA, The Cancer Genome Atlas.

with EGC, the scope was expanded to stage I/II. A total of 3 Asian patients with stage I/II gastric cancer were included in the present study. The clinicopathological features of the selected patients are shown in Table II. A total of 2,097 upregulated genes and 2,131 downregulated genes were identified, and are shown in a heat map and a volcano plot (Fig. 3A and B). Principal component analysis indicated that the gastric cancer tissue samples at stage I/II and their matched non-cancerous tissue samples could be divided into two groups based on the gene profile (Fig. 3C).

GO analysis, KEGG analysis and GSEA of DEGs. To explore the functions and pathways of DEGs in the cancer tissues and non-cancerous tissues of 3 Asian patients with stage I/II gastric cancer, GO analysis, KEGG analysis and GSEA were performed. Functional enrichment results revealed that DEGs were mainly associated with 'Regulation of membrane potential' and 'Muscle system process' in the biological process category. In the cellular component category, the DEGs were mainly enriched in 'Neuronal cell body', 'Collagen-containing extracellular matrix' and 'Cell-cell junction'. 'Channel activity' and 'Passive transmembrane transporter activity' were enriched terms in the molecular function (MF) category (Fig. 4A). In addition, KEGG pathway analysis indicated that the enrichment of DEGs was associated with 'Neuroactive ligand-receptor interaction' and 'Cytokine-cytokine receptor interaction'. 'Cell adhesion molecules (CAMs)' and 'Calcium signaling pathway' were 

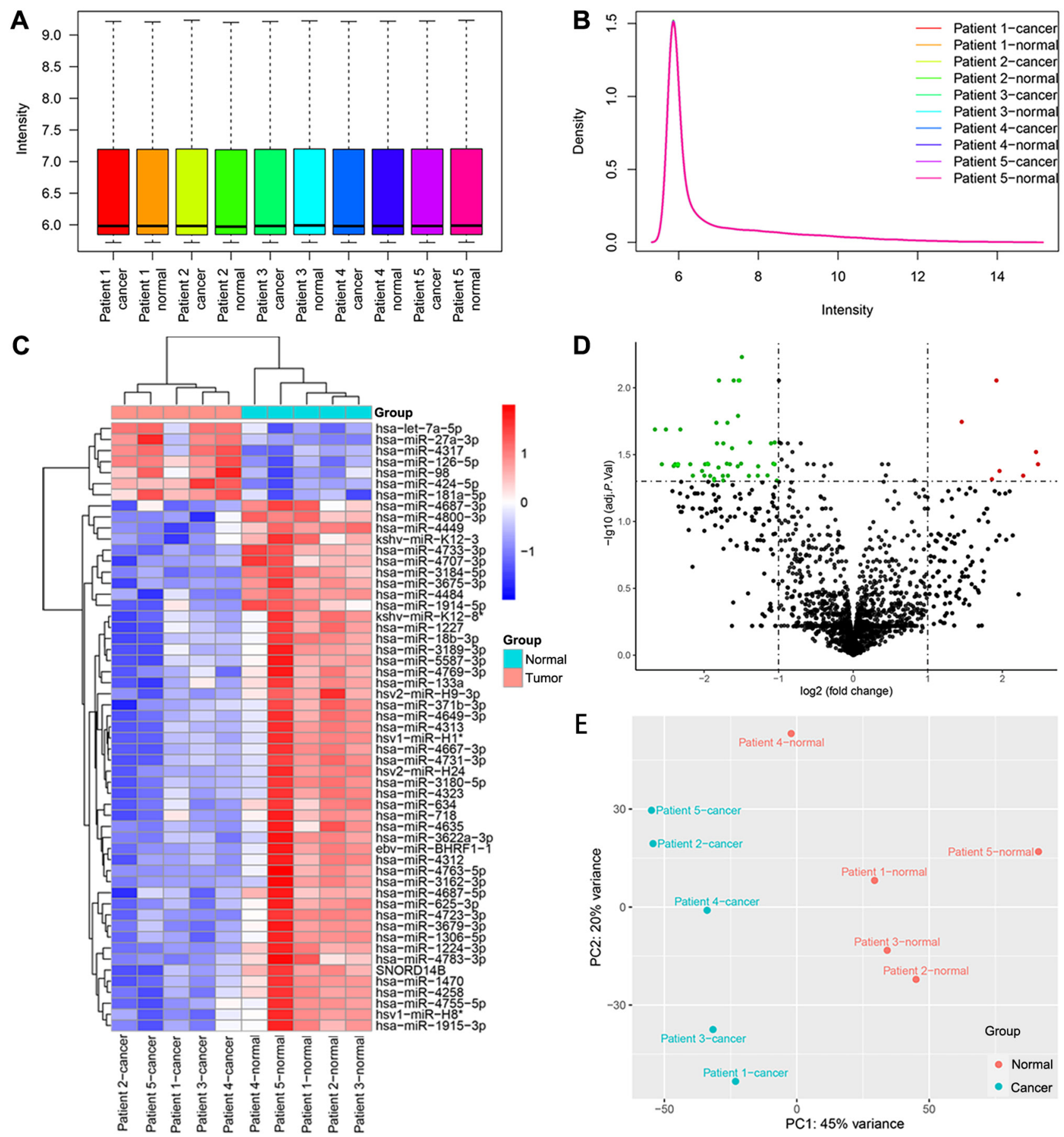

Figure 2. Data analysis of the miRNA microarray using five early gastric cancer tissues and five adjacent non-cancerous tissues, and the identification of DEMs. (A) Box plot after normalization, which shows the maximum, upper quartile, median, lower quartile and minimum of different datasets. (B) Density plot after normalization, which shows a probability distribution at different miRNA expression levels. (C) Heat map generated by unsupervised hierarchical clustering for DEMs between two groups. The color varies from red to blue, indicating upregulated or downregulated expression of DEMs, respectively. (D) Volcano plot of the DEMs in the miRNA microarray. Red and green dots represent upregulated and downregulated molecules (54 miRNAs and one small nuclear RNA), respectively. (E) Principal component analysis result of the tissue samples. adj. P. Val., adjusted P-value; DEMs, differentially expressed miRNAs; miRNA/miR, microRNA; PC, principal component.

also enriched (Fig. 4B). Finally, GSEA results suggested that pathways of the 'cell cycle', 'DNA replication', 'P53 signaling pathway' and 'natural killer cell-mediated cytotoxicity' tended to be activated in the cancer group (Fig. 4C-F).

miRNA-gene network construction and functions of target genes. After the DEMs and DEGs were identified, miRDB was used to predict the possible target relationships between them. For the upregulated miRNAs, target genes were predicted among the downregulated DEGs and vice versa. Subsequently, a miRNA-gene network was constructed (Fig. 5A).

GO and KEGG analyses of the predicted target genes were performed to learn more about the functions of the DEMs. In the MF category of GO analysis, the terms with the highest gene counts were 'Metal ion transmembrane 
A

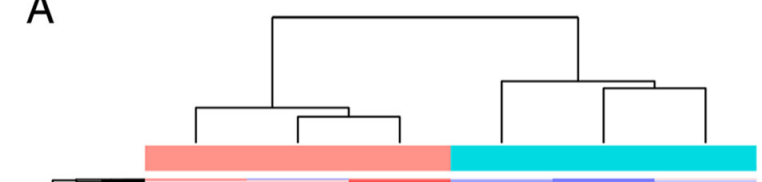

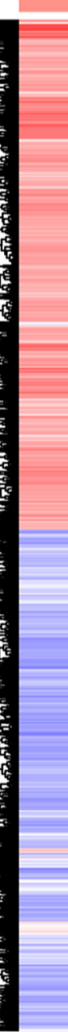
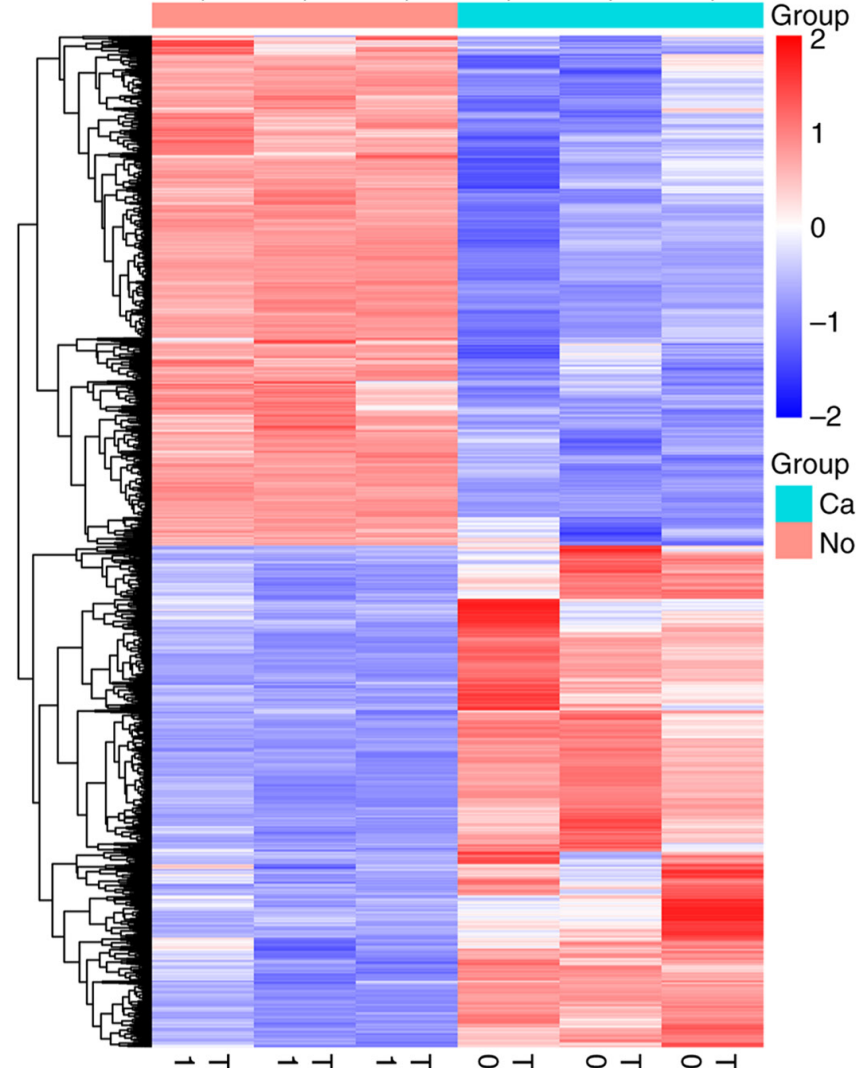
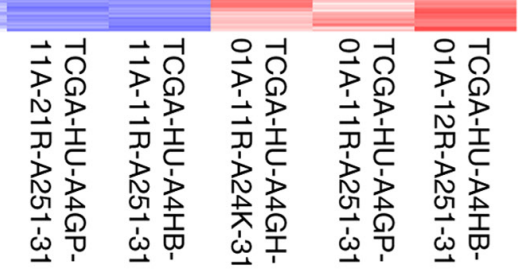

B
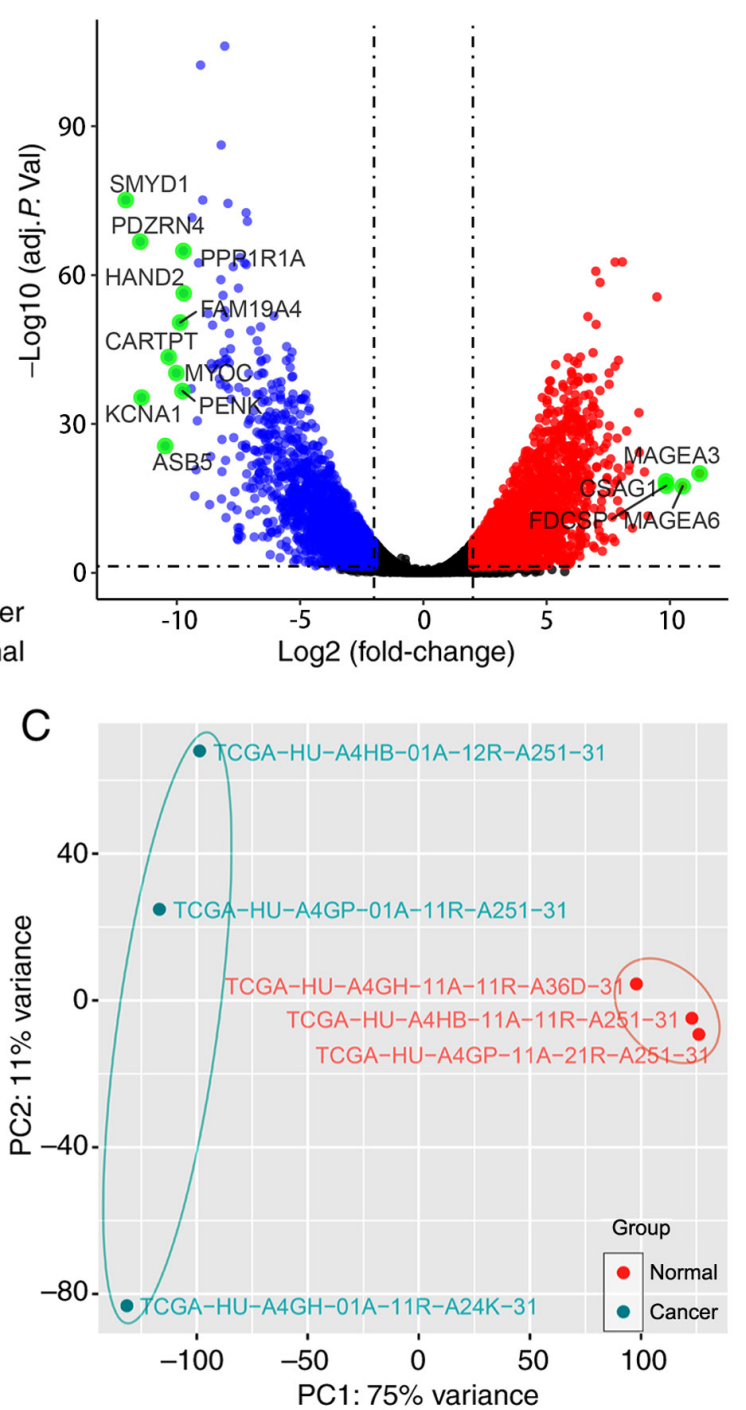

Figure 3. Gene expression profiles of three pairs of gastric cancer tissue samples and the adjacent normal mucosa tissue samples from 3 Asian patients with gastric cancer at stage I/II based on data obtained from TCGA. (A) Heat map generated by unsupervised hierarchical clustering for DEGs between the two groups. The color varies from red to blue, indicating upregulated or downregulated expression of DEGs, respectively. (B) Volcano plot of the DEGs. Red dots represent upregulated genes, and blue dots represent downregulated genes. Green dots represent genes with $\mid \log _{2}$ (fold change)l $\geq 9.5$ (C) Principal component analysis plot of the six samples. DEGs, differentially expressed genes; adj. P. Val., adjusted P-value; TCGA, The Cancer Genome Atlas; PC, principal component.

transporter activity', 'Actin binding' and 'Ion channel activity' (Fig. 5B). KEGG terms, such as 'Neuroactive ligand-receptor interaction', 'Pathways in cancer' and 'Focal adhesion', were significantly enriched (Fig. 5C).

The results of PPI analysis are shown in Fig. 5D. A total of 13 genes, including ADRB3, ADCYAP1, POMC, CALCB, PTHLH, ADCY5, GNB4, VIP, PTGDR, LHCGR, ADRB2, GPR83 and GIPR were filtered as hub genes in the network, which indicated that the DEMs may mainly work by regulating these hub genes.

Survival analysis validation. Clinical data and RNA expression data of Asian patients with gastric cancer (irrespective of the stage) from TCGA were used for survival analysis. A total of four miRNAs (hsa-let-7a-5p, hsa-miR-27a-3p, hsa-miR-126-5p and hsa-miR-424-5p) were identified to be significantly associated with the prognosis of patients with gastric cancer (Fig. 6).

\section{Discussion}

A total of seven upregulated and 40 downregulated human miRNAs were identified in EGC tissues compared with adjacent non-cancerous tissues. The functions of the DEMs were annotated using the TAM webtool. 'Hormones regulation' was the most enriched function term, which indicated that hormones may be regulated in EGC by miRNAs. A population-based matched cohort study in Sweden suggested that menopausal hormone therapy users are at a decreased risk of gastric adenocarcinoma (37). In addition, gonadotropin-releasing hormone (38), corticotropin-releasing hormone (39), steroid hormones (40) and growth hormone (41) have been reported to be associated with gastric cancer. However, more evidence is required to verify the role of hormone regulation in EGC.

Expression profiles of three pairs of cancer tissues and adjacent non-cancerous tissues from patients with 
Table III. Functional annotation of differentially expressed miRNAs using the tool for annotations of human miRNAs.

\begin{tabular}{|c|c|c|c|c|}
\hline Function term & Count & Percent & Fold & P-value \\
\hline Hormones regulation & 9 & 0.14516129 & 3.27217742 & 0.00062950 \\
\hline Human embryonic stem cell (hESC) regulation & 8 & 0.09411765 & 2.12156863 & 0.02291040 \\
\hline Immune response & 8 & 0.16666667 & 3.75694444 & 0.00052898 \\
\hline Inflammation & 8 & 0.19512195 & 4.39837398 & 0.00016338 \\
\hline Cell cycle related & 7 & 0.10606061 & 2.39078283 & 0.01856736 \\
\hline Epithelial-mesenchymal transition & 5 & 0.12195122 & 2.74898374 & 0.02820963 \\
\hline Cell death & 5 & 0.09090909 & 2.04924242 & 0.08525479 \\
\hline Hematopoiesis & 5 & 0.16129032 & 3.63575269 & 0.00867962 \\
\hline MiRNA tumor suppressors & 5 & 0.13513514 & 3.04617117 & 0.01854272 \\
\hline Lipid metabolism & 4 & 0.20000000 & 4.50833333 & 0.00898287 \\
\hline Angiogenesis & 3 & 0.12500000 & 2.81770833 & 0.08400821 \\
\hline Cell proliferation & 3 & 0.10714286 & 2.41517857 & 0.12100311 \\
\hline HIV latency & 3 & 0.14285714 & 3.22023810 & 0.06027038 \\
\hline Adipocyte differentiation & 3 & 0.11111111 & 2.50462963 & 0.11122976 \\
\hline Onco-miRNAs & 3 & 0.09677419 & 2.18145161 & 0.15217690 \\
\hline Apoptosis & 2 & 0.04545455 & 1.02462121 & 0.59774380 \\
\hline Bone regeneration & 2 & 0.05882353 & 1.32598039 & 0.45394525 \\
\hline Folliculogenesis & 2 & 0.28571429 & 6.44047619 & 0.03458783 \\
\hline Anti-cell proliferation (Hwang etal BJC2006) & 2 & 0.18181818 & 4.09848485 & 0.08125429 \\
\hline Cell division & 2 & 0.11764706 & 2.65196078 & 0.17103576 \\
\hline Immune system (Xiao's Cell2009) & 2 & 0.11111111 & 2.50462963 & 0.18736736 \\
\hline Cell differentiation & 1 & 0.05882353 & 1.32598039 & 0.54311212 \\
\hline Chemosensitivity of tumor cells & 1 & 0.25000000 & 5.63541667 & 0.16641762 \\
\hline DNA repair & 1 & 0.10000000 & 2.25416667 & 0.36724517 \\
\hline Granulopoiesis & 1 & 0.10000000 & 2.25416667 & 0.36724517 \\
\hline Carbohydrate metabolism & 1 & 0.14285714 & 3.22023810 & 0.27345270 \\
\hline Cell fate determination & 1 & 0.03846154 & 0.86698718 & 0.70138621 \\
\hline Heart development & 1 & 0.14285714 & 3.22023810 & 0.27345270 \\
\hline
\end{tabular}

miRNA, microRNA.

stage I/II gastric cancer were downloaded. Gastric cancer exhibits biological and epidemiological differences between Asian and non-Asian populations $(42,43)$. To improve the understanding of the roles of DEMs, the present study aimed to identify target mRNAs among DEGs in Asian patients. Since only 1 Asian patient with EGC has been included in the TCGA database to date, the scope was expanded to patients at stage I/II (AJCC, Eighth Edition). Notably, 'Neuroactive ligand-receptor interaction' and 'Calcium signaling pathway' were enriched KEGG terms for DEGs. 'Neuroactive ligand-receptor interaction', 'Axon guidance' and 'Neurotrophin signaling pathway' were enriched KEGG terms for the target genes of DEMs. Furthermore, 'muscle contraction' was an enriched biological process term in GO analysis of DEGs. These results suggest that pathways associated with nerves, muscle contraction and calcium signaling may serve a role in gastric cancer. There is much evidence regarding the association between the calcium signaling pathway and gastric cancer $(44,45)$. Regarding nerve and muscle contraction, certain proteins involved in neurodegenerative events are considered to be associated with gastric cancer, according to previous reports (46-48). Based on the target gene results, 'pathways in cancer' and other pathways associated with cancer, such as 'Focal adhesion' (49) and 'Melanoma', were also affected. Since the results obtained by this method are closely associated with the gene set, the two methods of detecting functional enrichment complement each other.

The miRNA-gene network was constructed. However, $>90 \%$ of the target genes were identified to be potentially modulated by hsa-let-7a-5p, hsa-miR-27a-3p, hsa-miR-126-5p, hsa-miR-424-5p, hsa-miR-181a-5p and hsa-miR-1915-3p in the network. Survival analysis of Asian patients demonstrated that four of the miRNAs (hsa-let-7a-5p, hsa-miR-27a-3p, hsa-miR-126-5p and hsa-miR-424-5p) were significantly associated with the prognosis of patients with gastric cancer. Multiple studies have demonstrated that hsa-miR-27a-3p (50-53) and hsa-miR-424-5p (54-56) are upregulated in gastric cancer and act as tumor activators. The present results demonstrated that hsa-miR-27a-3p and 
A

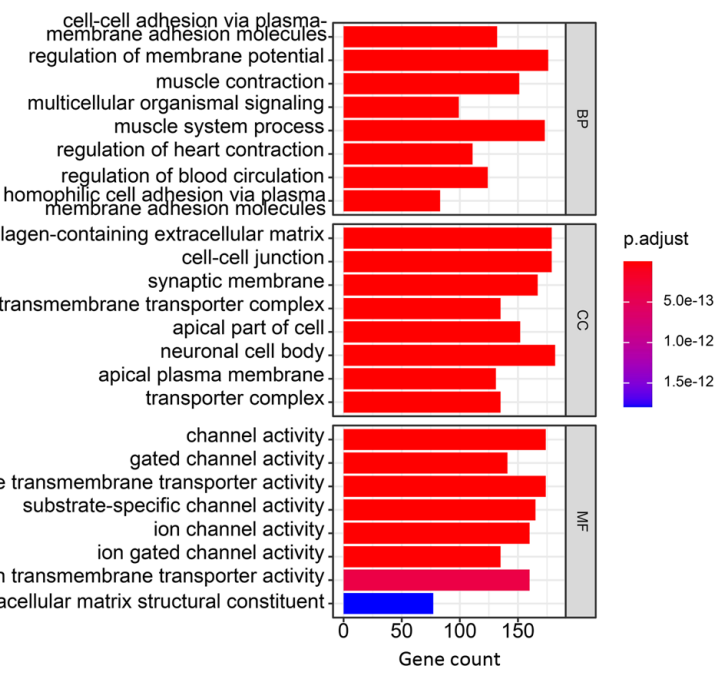

C
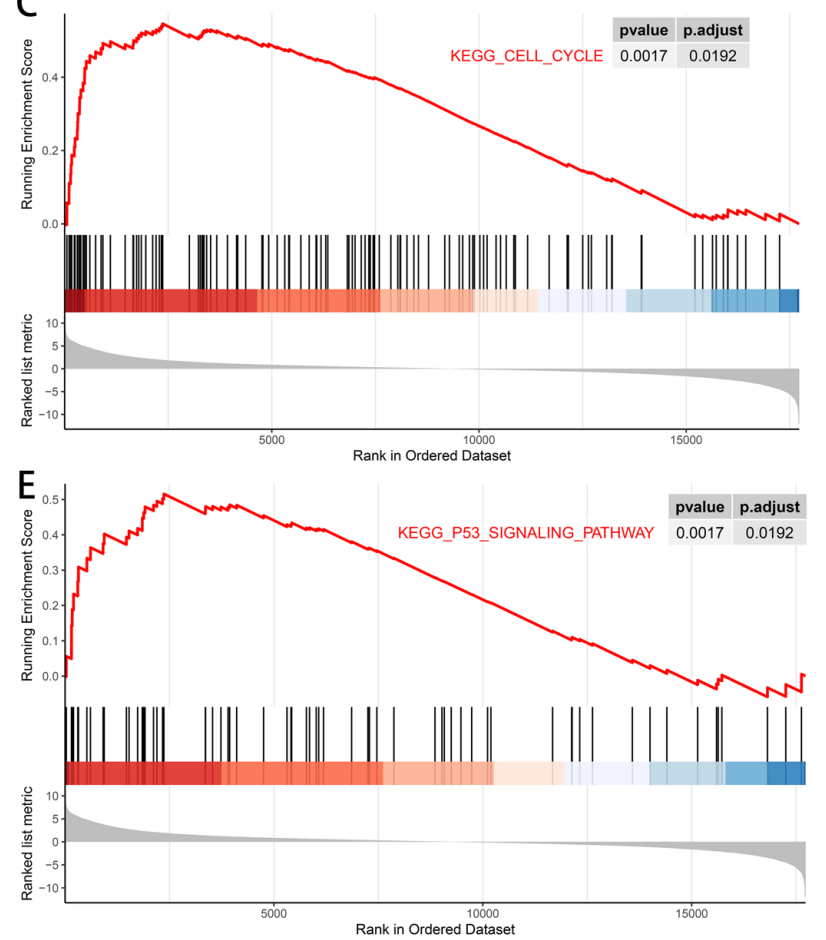

B

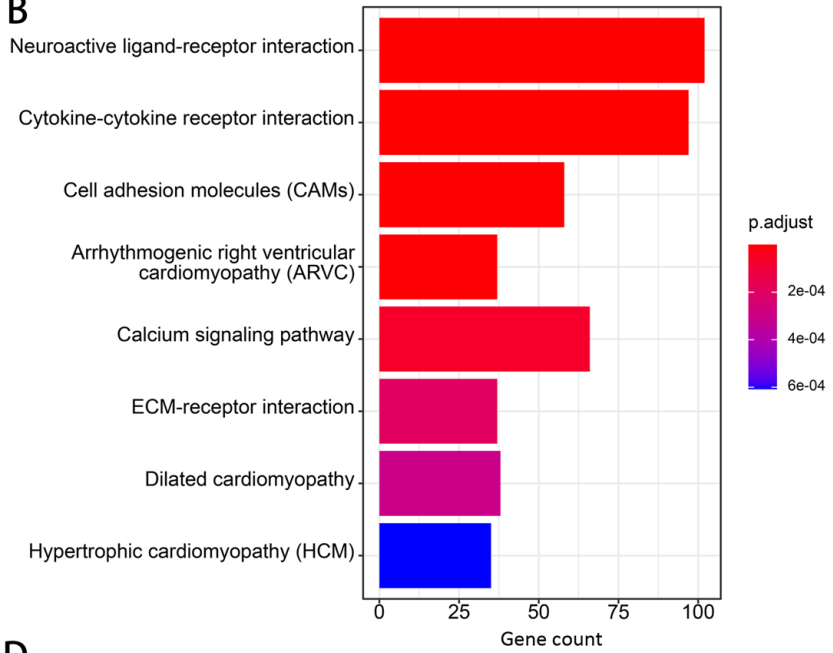

D

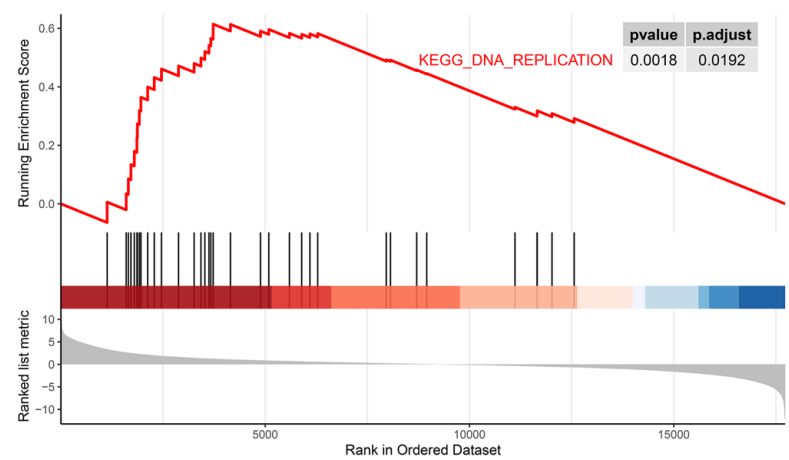

$\mathrm{F}$

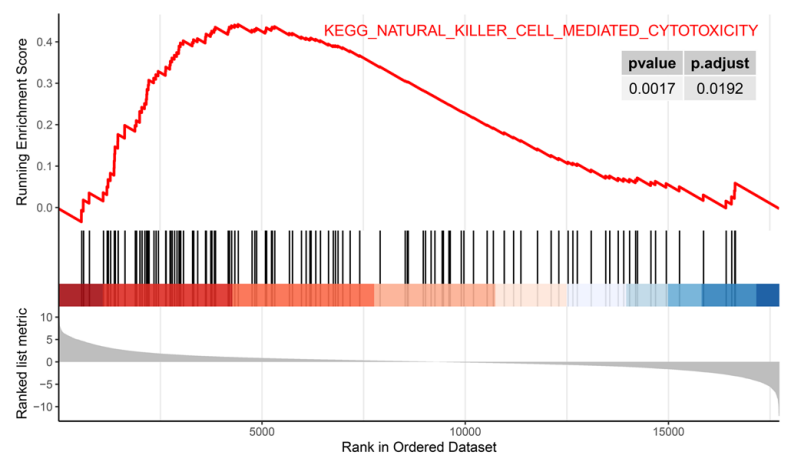

Figure 4. GO analysis, KEGG analysis and GSEA of the differentially expressed genes between the cancer tissues and non-cancerous tissues of 3 Asian patients with stage I/II gastric cancer. (A) Results of GO analysis, including BP, CC and MF aspects. (B) Results of KEGG analysis. (C-F) Results of GSEA revealing that the KEGG pathways of (C) 'cell cycle', (D) 'DNA replication', (E) 'P53 signaling pathway' and (F) 'natural killer cell-mediated cytotoxicity' were activated in the gastric cancer tissues at stage I/II. BP, biological process; CC, cellular component; GO, Gene Ontology; GSEA, Gene Set Enrichment Analysis; KEGG, Kyoto Encyclopedia of Genes and Genomes; MF, molecular function; p.adjust, adjusted P-value.

has-miR-424-5p could be upregulated in EGC and may affect the prognosis of patients with gastric cancer. Liang et al (57) reported that the hsa-let-7 family inhibits tumor invasion and metastasis by targeting myosin heavy chain 9 in human gastric cancer, which is different from the results of the present study. Since the sample size included in the present study was small, the results should be verified with larger sample sizes. To the best of our knowledge, there have been no studies on hsa-miR-126-5p in gastric cancer. Although a study using expression profile analysis of miRNAs in prostate cancer by next-generation sequencing demonstrated that hsa-miR-126-5p is highly expressed in tumor tissues (58), other studies came to different conclusions and have suggested that it may be a tumor suppressor $(59,60)$. The functions of
hsa-miR-126-5p in gastric cancer and EGC need to be verified. Experiments have demonstrated that hsa-miR-181a-5p can promote the progression of gastric cancer via Ras association domain family member 6-mediated mitogen activated kinase-like protein signaling activation (61) or by regulating protein tyrosine phosphatase non-receptor type 9 (62). Furthermore, hsa-miR-1915-3p inhibits Bcl-2 expression in the development of gastric cancer (63). hsa-miR-1915-3p serves a role in breast cancer inhibition (64) and increases drug sensitivity in colorectal cancer (65). The present results support the role of hsa-miR-181a-5p as a tumor activator and the role of hsa-miR-1915-3p as a tumor suppressor. Overall, the studies of miRNAs in EGC are still limited, and the present study provided some evidence for this. There are also 
A

PID1 ZNRF3 TMEM221 FGF18

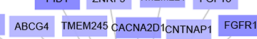

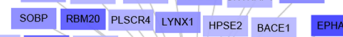

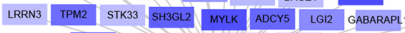

NOS1 MEOX2 KCNIP1 KY FRMPD1 DYNCIII RIMS3 NCS1

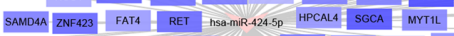

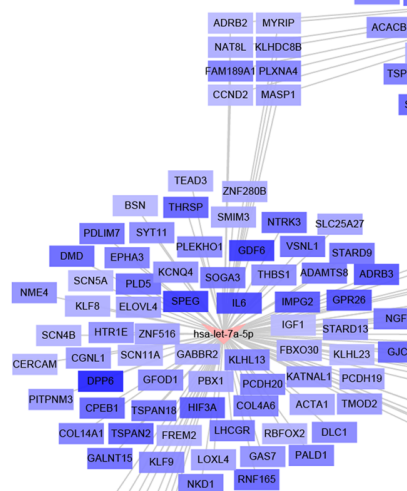

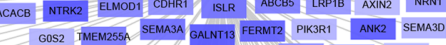

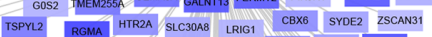

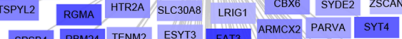

\begin{tabular}{|c|c|c|c|c|c|c|c|}
\hline SPSBA & RBM24 & TENM2 & ESYY13 & FAT3 ARMCX2 PARVA & SY14 \\
\hline SYT3 & RA998 COL24A1 & KCNAB1 & MKX AP1S2 \\
\hline
\end{tabular}

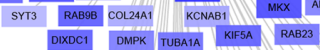

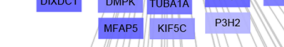

$\sqrt{\mathrm{DD} \times 3 \mathrm{Y}} \mathrm{KC}$

HDX

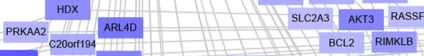

FOXP2 SEMAAC PEG10

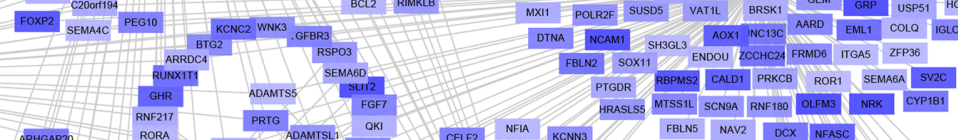

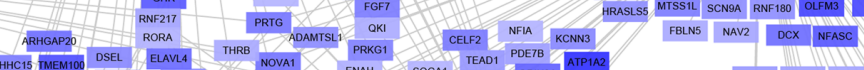

CA10 PDEBB ACTA2 SLC16A12 PTCHD1 JMY

CAMK1D CDK14 SFRP1 DOKG GLRA2 RAIYL ENOX1 CIPC

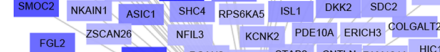

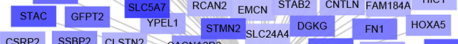

\begin{tabular}{lll} 
& \\
\hline
\end{tabular}

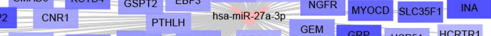

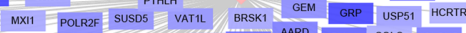

DTNA NCAM1 SHBCLI AOX1 NCL13C AARD EML1 COLO IC

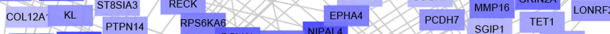

MTEF2 KCNMA1 TNANA
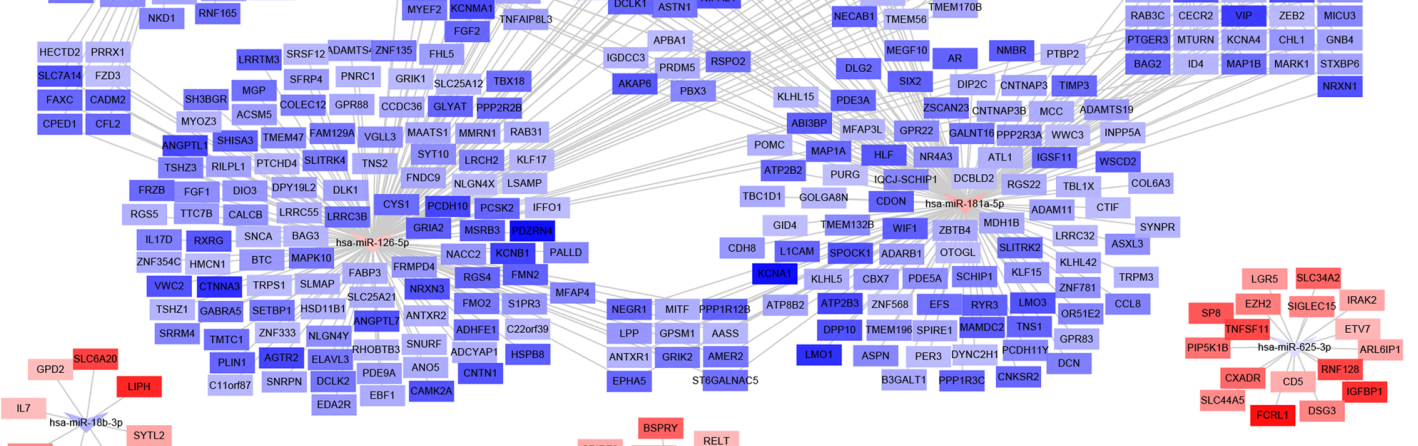

Noo1

NEK2 SHMT2

FGFR3

TRIM10 SP6 SLCSAS DERL3 RBBPENL

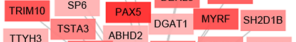

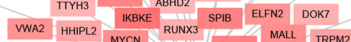

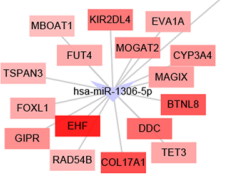

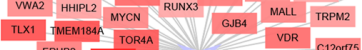

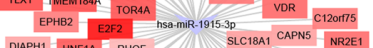
DIAPH1 HNF1A RHOF ADAMTS14 OVOL1 ZC3H12A FUT6 KRTBO PAORA SLCGAG SEMATA KRTCAPS IILRB PTPN6 FHDC1 TAGLN2 ANKRO22 KRTCAPF IILRE

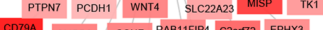
SMPD3 $/$ CCNF RABIFPA
CCR6 TREML2
YDJC

CGN

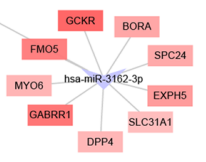

B

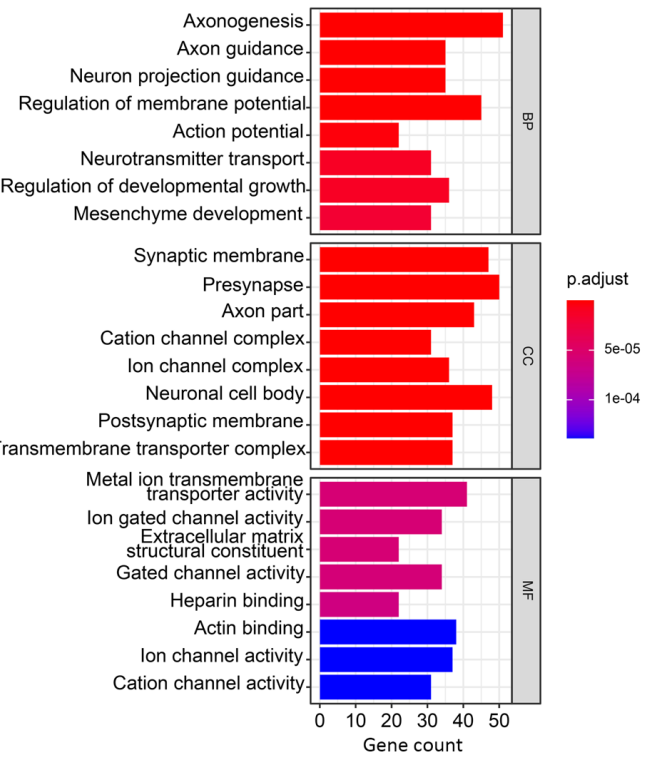

$\mathrm{C}_{\mathrm{Ne}}$

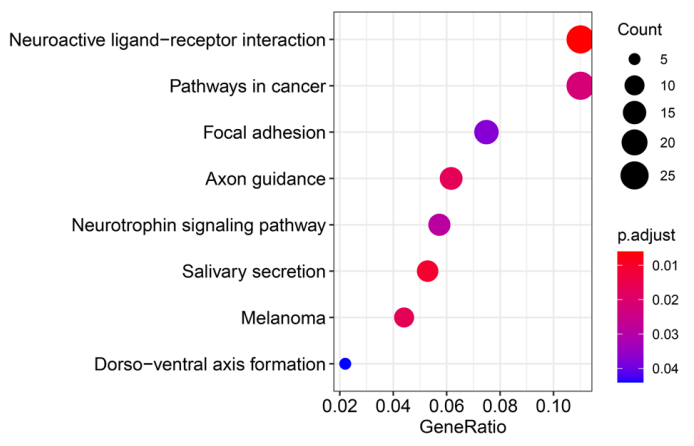

D

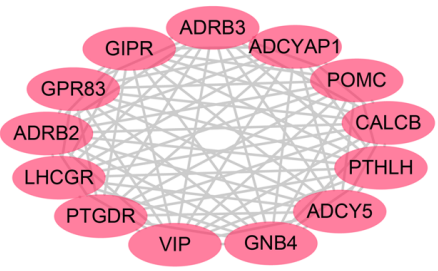

Figure 5. Construction of the miRNA-gene network and functions of the target genes. (A) Predicted miRNA-gene network in gastric cancer tissues at stage I/II. (B) Gene Ontology analysis and (C) Kyoto Encyclopedia of Genes and Genomes analysis of the predicted target genes of miRNAs. (D) Hub genes that interact closely with each other, which were filtered from the protein-protein interaction results. BP, biological process; CC, cellular component; FC, fold change; MF, molecular function; miRNA, microRNA; p.adjust, adjusted P-value.

differentially expressed viral miRNAs and snRNAs. Further research is required to clarify their functions.
The present study intended to perform preliminary screening of miRNAs and the results need to be verified in 
A

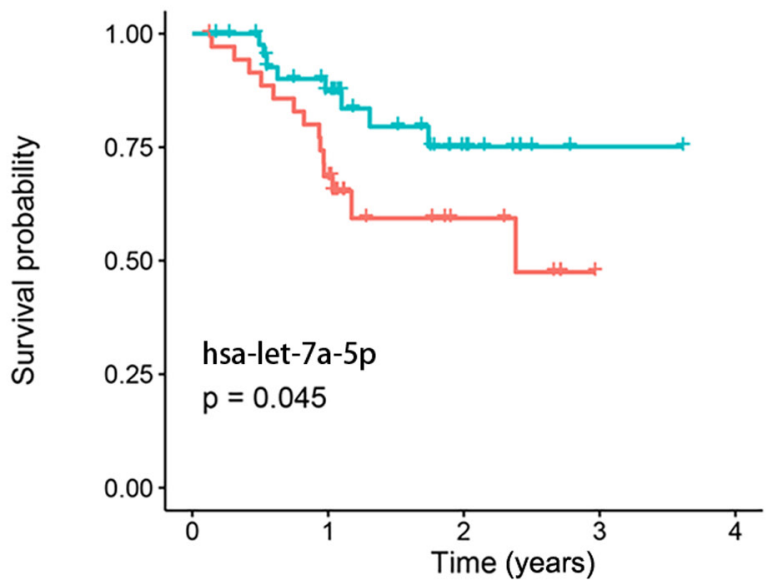

Number at risk

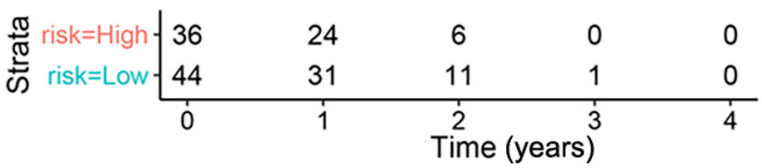

C

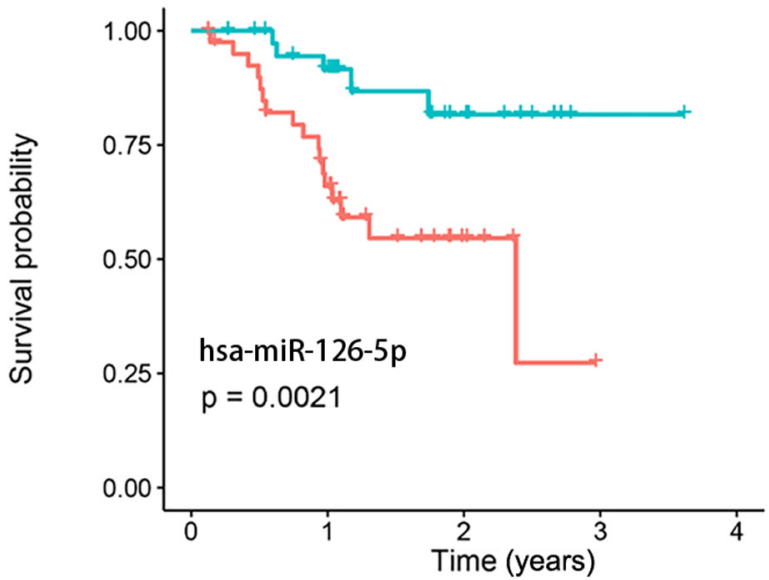

Number at risk

\begin{tabular}{|c|c|c|c|c|}
\hline \multirow{3}{*}{$\begin{array}{l}\frac{\pi}{\pi} \text { risk=High } \\
\text { ڤั) risk=Low }\end{array}$} & 41 & 24 & 5 & 0 \\
\hline & 39 & 31 & 12 & 1 \\
\hline & 0 & 1 & $\stackrel{2}{\text { Time }}$ & 3 \\
\hline
\end{tabular}

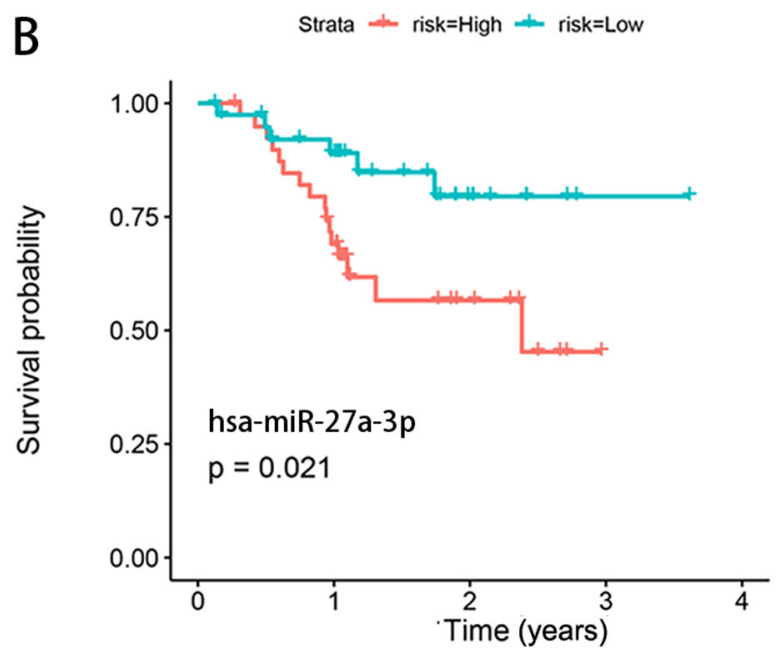

Number at risk

\begin{tabular}{|c|c|c|c|c|}
\hline \multirow{3}{*}{ 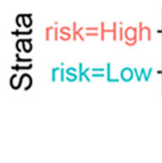 } & 40 & 26 & 8 & 0 \\
\hline & 40 & 29 & 9 & 1 \\
\hline & 0 & 1 & $\stackrel{2}{\operatorname{Tim}}$ & 3 \\
\hline
\end{tabular}

D

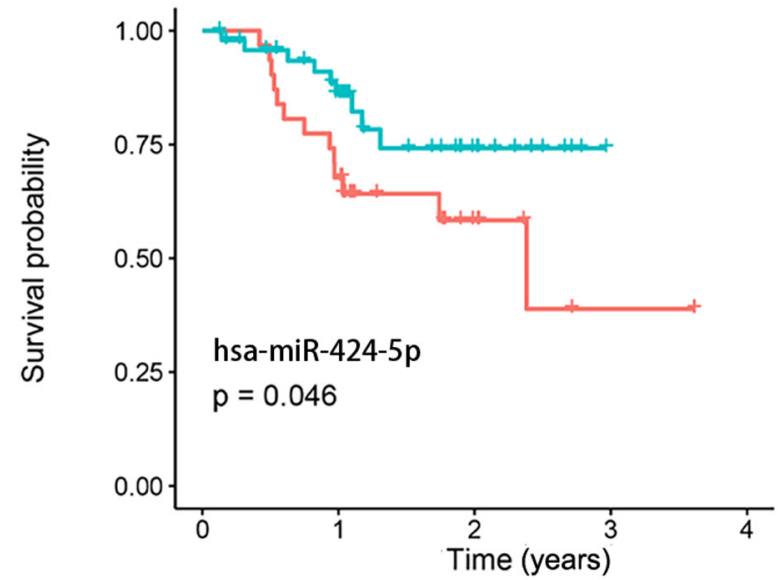

\section{Number at risk}

\begin{tabular}{|c|c|c|c|c|c|}
\hline$\frac{\pi}{\pi}$ risk $=$ High. & $\begin{array}{l}31 \\
49\end{array}$ & $\begin{array}{l}21 \\
34\end{array}$ & $\begin{array}{c}6 \\
11\end{array}$ & $\begin{array}{l}1 \\
0\end{array}$ & 0 \\
\hline & 0 & 1 & 2 & 3 & 4 \\
\hline
\end{tabular}

Figure 6. Survival analysis of the differentially expressed miRNAs. Prognostic value of (A) hsa-let-7a-5p, (B) hsa-miR-27a-3p, (C) hsa-miR-126-5p and (D) hsa-miR-424-5p in Asian patients with gastric cancer based on data from The Cancer Genome Atlas database. The x-axis indicates survival time in years, and the $y$-axis indicates survival probability. P-values, which are shown in each figure, refer to the comparison between the high and low miRNA expression groups, which were divided according to the best separation cutoff value. miR/miRNA, microRNA.

a larger cohort. Additionally, the present study was based on individuals in Gansu province and Asia, which is helpful to explore the particularity of gastric cancer in these regions. However, the population is also a limitation of the present study when the results need to be extended to other regions or other high incidence areas of gastric cancer. Comparing the results with other population cohorts should be a direction of future research.

In conclusion, in the present study, the miRNA profiles in five pairs of EGC tissues and adjacent non-cancerous tissues were investigated using a miRNA microarray. The possible mechanisms and abilities as prognostic factors of DEMs were assessed using bioinformatics methods. The present study revealed that certain miRNAs, including hsa-let-7a-5p, hsa-miR-27a-3p, hsa-miR-126-5p and hsa-miR-424-5p, were upregulated in EGC, and were associated with the prognosis of gastric cancer based on analysis of the miRNA expression profiles of patients from Gansu province, China. The present study could provide a basis for the identification of EGC screening biomarkers and for exploring the cause of the high incidence of gastric cancer in Gansu province, China from the perspective of miRNAs. 


\section{Acknowledgements}

Not applicable.

\section{Funding}

The present study was supported by the National Key R\&D Program of China (grant no. 2016YFC1302201), the Natural Science Foundation of Gansu Province, China (grant nos. 18JR3RA336 and 17JR5RA272) and the Fundamental Research Funds for the Central Universities (grant no. lzujbky-2020-kb16).

\section{Availability of data and materials}

The datasets generated and analyzed during the current study are available in the Gene Expression Omnibus repository (https://www.ncbi.nlm.nih.gov/geo/query/acc. cgi?\&acc=GSE158315).

\section{Authors' contributions}

YL, WS and YW conceived the study, and YZ and LL designed the study. YZ, HW and HY were involved in the sample and data collection. YL, LL and TH performed the RNA extraction experiments and the quality control. YL, LL and $\mathrm{HW}$ performed the bioinformatics analysis and prepared the figures. YZ, HZ, HY and XC performed the statistical analysis. YL, YZ and TH drafted the manuscript. HZ, XC and WS contributed substantially to the revision of the manuscript. The authenticity of all the raw data has been assessed by YL, WS and YW. All authors read and approved the final manuscript.

\section{Ethics approval and consent to participate}

The study protocol was approved by the Ethics Committee of The First Hospital of Lanzhou University (approval no. LDYYLL2012001; Lanzhou, China). Written informed consent forms were signed by the patients and their family members.

\section{Patient consent for publication}

Not applicable.

\section{Competing interests}

The authors declare that they have no competing interests.

\section{References}

1. Bray F, Ferlay J, Soerjomataram I, Siegel RL, Torre LA and Jemal A: Global cancer statistics 2018: GLOBOCAN estimates of incidence and mortality worldwide for 36 cancers in 185 countries. CA Cancer J Clin 68: 394-424, 2018.

2. Liu Y, Zhang X, Chen L, Zhao Q and Xia X: Cancer incidence and mortality in Gansu province, 2012. Chin J Cancer Res 28: 301-310, 2016.

3. Chen W, Zheng R, Zhang S, Zeng H, Zuo T, Jia M, Xia C, Zou X and He J: Report of cancer incidence and mortality in China, 2012. China Cancer 25: 1-8, 2016.
4. Guo Q, Liu X, Lu L, Yuan H, Wang Y, Chen Z, Ji R and Zhou Y: Comprehensive evaluation of clinical efficacy and safety of celecoxib combined with chemotherapy in management of gastric cancer. Medicine (Baltimore) 96: e8857, 2017.

5. Ji R: Gastric cancer screening and related risk factors analysis in the Wuwei Natrual population cohort. Lanzhou University, 2018.

6. Pasechnikov V, Chukov S, Fedorov E, Kikuste I and Leja M: Gastric cancer: Prevention, screening and early diagnosis. World J Gastroenterol 20: 13842-13862, 2014.

7. Everett SM and Axon AT: Early gastric cancer in Europe. Gut 41: 142-150, 1997.

8. Suzuki H, Oda I, Abe S, Sekiguchi M, Mori G, Nonaka S, Yoshinaga S and Saito Y: High rate of 5-year survival among patients with early gastric cancer undergoing curative endoscopic submucosal dissection. Gastric Cancer 19: 198-205, 2016.

9. Crew KD and Neugut AI: Epidemiology of gastric cancer. World J Gastroenterol 12: 354-362, 2006.

10. Gong W, Luo S, Hu R, Wang H, Pan J, Fei F, Wu H and Yu M: Survival analysis of gastric cancer patients during 2005-2010 in Zhejiang Province, China. Zhonghua Zhong Liu Za Zhi 36: 636-639, 2014 (In Chinese).

11. Zheng L, Wu C, Xi P, Zhu M, Zhang L, Chen S, Li X, Gu J and Zheng Y: The survival and the long-term trends of patients with gastric cancer in Shanghai, China. BMC Cancer 14: 300, 2014.

12. Hamashima C, Shabana M, Okada K, Okamoto M and Osaki Y: Mortality reduction from gastric cancer by endoscopic and radiographic screening. Cancer Sci 106: 1744-1749, 2015.

13. So JBY, Kapoor R, Zhu F, Koh C, Zhou L, Zou R, Tang YC, Goo PCK, Rha SY, Chung HC, et al: Development and validation of a serum microRNA biomarker panel for detecting gastric cancer in a high-risk population. Gut 70: 829-837, 2021.

14. Bartel DP: MicroRNAs: Genomics, biogenesis, mechanism, and function. Cell 116: 281-297, 2004.

15. Calin GA, Sevignani C, Dumitru CD, Hyslop T, Noch E, Yendamuri S, Shimizu M, Rattan S, Bullrich F, Negrini M and Croce CM: Human microRNA genes are frequently located at fragile sites and genomic regions involved in cancers. Proc Natl Acad Sci USA 101: 2999-3004, 2004

16. Han J, Lee Y, Yeom KH, Kim YK, Jin H and Kim VN: The Drosha-DGCR8 complex in primary microRNA processing. Genes Dev 18: 3016-3027, 2004.

17. Zheng GD, Xu ZY, Hu C, Lv H, Xie XH, Huang T, Zhang YQ, Chen GP, Fu YF and Cheng XD: Exosomal miR-590-5p in serum as a biomarker for the diagnosis and prognosis of gastric cancer front. Mol Biosci 8: 636566, 2021.

18. Shi Y, Wang Z, Zhu X, Chen L, Ma Y, Wang J, Yang X and Liu Z: Exosomal miR-1246 in serum as a potential biomarker for early diagnosis of gastric cancer. Int J Clin Oncol 25: 89-99, 2020.

19. Xia L, Zhang D, Du R, Pan Y, Zhao L, Sun S, Hong L, Liu J and Fan D: miR-15b and miR-16 modulate multidrug resistance by targeting BCL2 in human gastric cancer cells. Int J Cancer 123: 372-379, 2008.

20. Shin VY and Chu KM: MiRNA as potential biomarkers and therapeutic targets for gastric cancer. World J Gastroenterol 20: 10432-10439, 2014

21. Lu M, Hu C, Wu F, Shu L, Pan Y, Liu X, Liu P, Ma F, Deng C and Huang M: MiR-320a is associated with cisplatin resistance in lung adenocarcinoma and its clinical value in non-small cell lung cancer: A comprehensive analysis based on microarray data. Lung Cancer 147: 193-197, 2020.

22. Amin MB, Edge SB, Greene FI, Byrd DR, Brookland RK, Washington MK, Gershenwald JE, Compton CC, Hess KR, Sullivan DC, et al: AJCC cancer staging manual. Springer-Verlag. New York, the United States, 2016.

23. Ritchie ME, Phipson B, Wu D, Hu Y, Law CW, Shi W and Smyth GK: Limma powers differential expression analyses for RNA-sequencing and microarray studies. Nucleic Acids Res 43: e47, 2015

24. Barrett T, Wilhite SE, Ledoux P, Evangelista C, Kim IF, Tomashevsky M, Marshall KA, Phillippy KH, Sherman PM, Holko M, et al: NCBI GEO: Archive for functional genomics data sets-update. Nucleic Acids Res 41: D991-D995, 2013.

25. Li J, Han X, Wan Y, Zhang S, Zhao Y, Fan R, Cui Q and Zhou Y: TAM 2.0: Tool for MicroRNA set analysis. Nucleic Acids Res 46: W180-W185, 2018.

26. Love MI, Huber W and Anders S: Moderated estimation of fold change and dispersion for RNA-seq data with DESeq2. Genome Biol 15: 550, 2014

27. Gaudet P and Dessimoz C: Gene ontology: Pitfalls, biases, and remedies. Methods Mol Biol 1446: 189-205, 2017. 
28. Kanehisa M, Furumichi M, Tanabe M, Sato Y and Morishima K KEGG: New perspectives on genomes, pathways, diseases and drugs. Nucleic Acids Res 45: D353-D361, 2017.

29. Subramanian A, Tamayo P, Mootha VK, Mukherjee S, Ebert BL, Gillette MA, Paulovich A, Pomeroy SL, Golub TR, Lander ES and Mesirov JP: Gene set enrichment analysis: A knowledge-based approach for interpreting genome-wide expression profiles. Proc Natl Acad Sci USA 102: 15545-15550, 2005

30. Yu G, Wang LG, Han Y and He QY: clusterProfiler: An $\mathrm{R}$ package for comparing biological themes among gene clusters. OMICS 16: 284-287, 2012.

31. Wickham H: ggplot2: Elegant Graphics for Data Analysis. Springer-Verlag, New York, NY, 2016.

32. Chen Y and Wang X: miRDB: An online database for prediction of functional microRNA targets. Nucleic Acids Res 48: D127-D131, 2020.

33. Shannon P, Markiel A, Ozier O, Baliga NS, Wang JT, Ramage D, Amin N, Schwikowski B and Ideker T: Cytoscape: A software environment for integrated models of biomolecular interaction networks. Genome Res 13: 2498-2504, 2003.

34. Szklarczyk D, Gable AL, Lyon D, Junge A, Wyder S, Huerta-Cepas J, Simonovic M, Doncheva NT, Morris JH, Bork P, et al: STRING v11: Protein-protein association networks with increased coverage, supporting functional discovery in genome-wide experimental datasets. Nucleic Acids Res 47: D607-D613, 2019.

35. Bader GD and Hogue CW: An automated method for finding molecular complexes in large protein interaction networks. BMC Bioinformatics 4: 2, 2003.

36. Therneau TM and Grambsch PM: Modeling Survival Data: Extending the Cox Model. Springer-Verlag, New York, NY, 2000

37. Brusselaers N, Maret-Ouda J, Konings P, El-Serag HB and Lagergren J: Menopausal hormone therapy and the risk of esophageal and gastric cancer. Int J Cancer 140: 1693-1699, 2017.

38. Lu M, Zhu J, Ling Y, Shi W, Zhang C and Wu H: The lower expression of gonadotropin-releasing hormone receptor associated with poor prognosis in gastric cancer. Int J Clin Exp Med 8: 13365-13370, 2015.

39. Yang S, Liu W, Wen J, Zhu M and Xu S: Corticotropin releasing hormone is correlated with tumorigenesis of gastric cancer. Cancer Invest 31: 167-171, 2013.

40. Frycz BA, Murawa D, Borejsza-Wysocki M, Wichtowski M, Spychała A, Marciniak R, Murawa P, Drews M and Jagodziński PP: mRNA expression of steroidogenic enzymes, steroid hormone receptors and their coregulators in gastric cancer. Oncol Lett 13: 3369-3378, 2017.

41. Gan J, Ke X, Jiang J, Dong H, Yao Z, Lin Y, Lin W, Wu X, Yan S, Zhuang Y, et al: Growth hormone-releasing hormone receptor antagonists inhibit human gastric cancer through downregulation of PAK1-STAT3/NF- $\mathrm{B}$ signaling. Proc Natl Acad Sci USA 113 14745-14750, 2016.

42. Yamada T, Yoshikawa T, Taguri M, Hayashi T, Aoyama T, Sue-Ling HM, Bonam K, Hayden JD and Grabsch HI: The survival difference between gastric cancer patients from the UK and Japan remains after weighted propensity score analysis considering all background factors. Gastric Cancer 19: 479-489, 2016.

43. Kerckhoffs KGP,Liu DHW, SaragoniL, van der PostRS, Langer R, Bencivenga M, Iglesias M, Gallo G, Hewitt LC, Fazzi GE, et al: Mucin expression in gastric- and gastro-oesophageal signet-ring cell cancer: Results from a comprehensive literature review and a large cohort study of Caucasian and Asian gastric cancer. Gastric Cancer 23: 765-779, 2020.

44. Zhang ZL, Li ZR, Li JS and Wang SR: Calcium-sensing receptor antagonist NPS-2143 suppresses proliferation and invasion of gastric cancer cells. Cancer Gene Ther 27: 548-557, 2020

45. Tang B, Wu J, Zhu MX, Sun X, Liu J, Xie R, Dong TX, Xiao Y, Carethers JM, Yang S and Dong H: VPAC1 couples with TRPV4 channel to promote calcium-dependent gastric cancer progression via a novel autocrine mechanism. Oncogene 38: 3946-3961, 2019.

46. Ge C, Li Q, Wang $\mathrm{L}$ and $\mathrm{Xu} \mathrm{X}$ : The role of axon guidance factor semaphorin $6 \mathrm{~B}$ in the invasion and metastasis of gastric cancer. J Int Med Res 41: 284-292, 2013.

47. Pan G, Zhang X, Ren J, Lu J, Li W, Fu H, Zhang S and Li J: Semaphorin 5A, an axon guidance molecule, enhances the invasion and metastasis of human gastric cancer through activation of MMP9. Pathol Oncol Res 19: 11-18, 2013.
48. Park T, Lee YJ, Jeong SH, Choi SK, Jung EJ, Ju YT, Jeong CY, Park M, Hah YS, Yoo J, et al: Overexpression of neuron-specific enolase as a prognostic factor in patients with gastric cancer. J Gastric Cancer 17: 228-236, 2017.

49. Eke I and Cordes N: Focal adhesion signaling and therapy resistance in cancer. Semin Cancer Biol 31: 65-75, 2015.

50. Du M, Zheng R, Ma G, Chu H, Lu J, Li S, Xin J, Tong N, Zhang G, Wang W, et al: Remote modulation of IncRNA GCLET by risk variant at $16 \mathrm{p} 13$ underlying genetic susceptibility to gastric cancer. Sci Adv 6: eaay5525, 2020.

51. Yang F, Chen X, Li X, Chen J, Tang Y, Cai Y, Wang Y, Chen Z, Li L, Li R and Deng Z: Long intergenic non-protein coding RNA 1089 suppresses cell proliferation and metastasis in gastric cancer by regulating miRNA-27a-3p/epithelial-mesenchymal transition (EMT) axis. Cancer Manag Res 12: 5587-5596, 2020.

52. Geng G, Liu X, Xu A, Lu Z, Chen K, He J, Qi D and Yuan X: Low abundance of TFPI-2 by both promoter methylation and miR-27a-3p regulation is linked with poor clinical outcome in gastric cancer. J Gene Med 22: e3166, 2020.

53. Zhou L, Liang X, Zhang L, Yang L, Nagao N, Wu H, Liu C, Lin S, Cai G and Liu J: MiR-27a-3p functions as an oncogene in gastric cancer by targeting BTG2. Oncotarget 7: 51943-51954, 2016.

54. Zhang J, Liu H, Hou L, Wang G, Zhang R, Huang Y, Chen X and Zhu J: Circular RNA_LARP4 inhibits cell proliferation and invasion of gastric cancer by sponging miR-424-5p and regulating LATS1 expression. Mol Cancer 16: 151, 2017.

55. Wei S, Li Q, Li Z, Wang L, Zhang L and Xu Z: Correction: miR-424-5p promotes proliferation of gastric cancer by targeting Smad3 through TGF- $\beta$ signaling pathway. Oncotarget 8: 34018 , 2017.

56. Wei S, Li Q, Li Z, Wang L, Zhang L and Xu Z: miR-424-5p promotes proliferation of gastric cancer by targeting Smad3 through TGF- $\beta$ signaling pathway. Oncotarget 7: 75185-75196, 2016.

57. Liang S, He L, Zhao X, Miao Y, Gu Y, Guo C, Xue Z, Dou W, $\mathrm{Hu} \mathrm{F}$, Wu K, et al: MicroRNA let-7f inhibits tumor invasion and metastasis by targeting MYH9 in human gastric cancer. PLoS One 6: e18409, 2011.

58. Song C, Chen H, Wang T, Zhang W, Ru G and Lang J: Expression profile analysis of microRNAs in prostate cancer by next-generation sequencing. Prostate 75: 500-516, 2015.

59. Queiroz AL, Zhang B, Comstock DE, Hao Y, Eriksson M, Hydbring P, Vakifahmetoglu-Norberg $\mathrm{H}$ and Norberg E: miR-126-5p targets Malate Dehydrogenase 1 in non-small cell lung carcinomas. Biochem Biophys Res Commun 499: 314-320, 2018.

60. Wang C, Zhou B, Liu M, Liu Y and Gao R: miR-126-5p restoration promotes cell apoptosis in cervical cancer by targeting Bcl212. Oncol Res 25: 463-470, 2017.

61. Mi Y, Zhang D, Jiang W, Weng J, Zhou C, Huang K, Tang H, Yu Y, Liu X, Cui W, et al: miR-181a-5p promotes the progression of gastric cancer via RASSF6-mediated MAPK signalling activation. Cancer Lett 389: 11-22, 2017.

62. Liu Z, Sun F, Hong Y, Liu Y, Fen M, Yin K, Ge X, Wang F, Chen X and Guan W: MEG2 is regulated by miR-181a-5p and functions as a tumour suppressor gene to suppress the proliferation and migration of gastric cancer cells. Mol Cancer 16: 133, 2017.

63. Cui HW, Han WY,Hou LN, Yang L, Li X and Su XL: miR-1915-3p inhibits Bcl-2 expression in the development of gastric cancer. Biosci Rep 39: BSR20182321, 2019.

64. Jin ML, Kim YW, Jin HL, Kang H, Lee EK, Stallcup MR and Jeong KW: Aberrant expression of SETD1A promotes survival and migration of estrogen receptor $\alpha$-positive breast cancer cells. Int J Cancer 143: 2871-2883, 2018.

65. Xu K, Liang X, Cui D, Wu Y, Shi W and Liu J: miR-1915 inhibits Bcl-2 to modulate multidrug resistance by increasing drug-sensitivity in human colorectal carcinoma cells. Mol Carcinog 52: 70-78, 2013.

This work is licensed under a Creative Commons Attribution-NonCommercial-NoDerivatives 4.0 International (CC BY-NC-ND 4.0) License. 